\title{
The effect of the dispersion of microfibrillated cellulose on the mechanical properties of melt-compounded polypropylene-polyethylene copolymer
}

\author{
Caterina Palange $\cdot$ Marcus A. Johns • David J. Scurr • Jonathan S. Phipps • \\ Stephen J. Eichhorn
}

Received: 28 June 2019/Accepted: 12 September 2019/Published online: 27 September 2019

(C) The Author(s) 2019

\begin{abstract}
Microfibrillated cellulose (MFC) is a highly expanded, high surface area networked form of cellulose-based reinforcement. Due to the poor compatibility of cellulose with most common apolar thermoplastic matrices, the production of cellulosereinforced composites in industry is currently limited to polar materials. In this study, a facile water-based chemistry, based on the reaction of MFC with tannic acid and subsequent functionalisation with an alkyl amine, is used to render the surface of the MFC fibrils hydrophobic and enhance the dispersion of the cellulose-based filler into an apolar thermoplastic matrix. The level of dispersion of the compatibilized MFC reinforced composites was evaluated using Time of Flight Secondary Ion Mass Spectrometry and multi-
\end{abstract}

Electronic supplementary material The online version of this article (https://doi.org/10.1007/s10570-019-02756-8) contains supplementary material, which is available to authorized users.

C. Palange $\cdot$ J. S. Phipps

FiberLean Technologies Ltd., Par Moor Centre, Par Moor Rd, Par PL24 2SQ, UK

C. Palange $\cdot$ M. A. Johns $\cdot$ S. J. Eichhorn ( $\square)$

School of Civil, Aerospace, and Mechanical Engineering, Bristol Composites Institute (ACCIS), University Walk, University of Bristol, Bristol BS8 1TR, UK

e-mail: s.j.eichhorn@bristol.ac.uk

\section{J. Scurr}

School of Pharmacy, University of Nottingham, Boots

Science Building, Nottingham NG7 2RD, UK channel Spectral Confocal Laser Scanning Microscopy. The agglomeration of cellulosic filler within the composites was reduced by functionalising the surface of the MFC fibrils with tannic acid and octadecylamine. The resulting composites exhibited an increase in modulus at a high cellulose content. Despite the dispersion of a large portion of the functionalised filler, the presence of some remaining aggregates affected the impact properties of the composites produced.

Keywords Microfibrillated cellulose $\cdot$ Composites · Mechanical properties

\section{Introduction}

Cellulose-based nanofillers have the potential to increase the mechanical performance of composites dramatically, even at extremely low concentrations (Duchemin et al. 2009; Spoljaric et al. 2009; Miao and Hamad 2013; Pöllänen et al. 2013; Lee et al. 2014). The production of composites based on cellulose fillers and polyolefins has also been the target of much recent research (Peijs et al. 1998; Wambua et al. 2003; Ljungberg et al. 2006; Guo et al. 2013). Nanomaterials are defined as a class of materials having at least one dimension less than $100 \mathrm{~nm}$ (Siro et al. 2010). Nanofillers, one form of nanomaterials, are generally characterised by an extremely high surface to volume 
ratio, which generates an extended filler-matrix interfacial area (Siro et al. 2010; Sehaqui et al. 2011). Using an appropriate filler-matrix combination, it is possible to obtain reinforced nanocomposites in which the strong and extended interfacial area increases the mechanical properties of the matrix (Klemm et al. 2011; Missoum et al. 2013; Khalil et al. 2014).

Microfibrillated cellulose (MFC) is characterized by a fibrillar network morphology and a highly expanded interfacial area alongside attractive mechanical properties (calculated Young's modulus of $\approx 20 \mathrm{GPa}$ (network) and strength of $\approx 240 \mathrm{MPa}$ ) (Zimmermann et al. 2004, 2005; Leitner et al. 2007), low production cost, renewability and wide availability (Herrick et al. 1983; Klemm et al. 2006; Henriksson and Berglund 2007; Iwamoto et al. 2007; Siro et al. 2010; Spence et al. 2011). These characteristics render MFC an interesting nano-reinforcement. The structure and characteristics of MFC are dependent on the source of the raw material and on the fibrillation process. The MFC used in this study was produced at FiberLean Technologies Ltd.; it is uncharged, due to the mechanical production method used, and hydrophilic due to the presence of the hydroxyl groups on the surface of the fibrils. Polyolefins represent a large portion of the polymer market with a global annual production of 135 million tonnes (Woodhams et al. 1984; Malkapuram et al. 2008), and have a wellestablished industrial production route, from synthesis up to the final product conveyance. The products obtained from polyolefins are durable, chemically stable, have low melting temperatures and viscosities and excellent processability. The efficient production of MFC-reinforced polyolefins represents an important step in the establishment of naturally derived composites. Unfortunately, an industrial method to produce MFC-reinforced composites using the normal polyolefin manufacturing process has not yet been developed. The main issue to solve with these composites is the incompatibility of untreated cellulose fillers with hydrophobic polymer matrices. This incompatibility leads to a weak filler-matrix interface, and thereby poorly performing composites. Cellulosic fillers also possess a strong tendency to agglomerate, thereby minimising the surface exposed to the unfavourable environment represented by the apolar matrix.

Chemical surface modification (Gruber and Granzow 1996; Heux et al. 2000; Bonini et al. 2002; Hafrén et al. 2006) can substitute the hydroxyl groups on the surface of MFC fibrils. This decreases surface energy (Klemm et al. 2005; Maya and Rajesh 2008; Maya and Sabu 2008), potentially improving the mixing and dispersion of the filler and preventing aggregation (Habibi et al. 2010). Chemical treatments on the surface of MFC fibrils can be divided into those based on organic solvents and water-based systems. Organic solvent-based techniques are impractical due to the large volume of chemicals required to treat small amounts of MFC (Kazayawoko et al. 1997; Matias et al. 2000). Nevertheless, the functionalisation of cellulose with maleic anhydride grafted polypropylene (MAgPP) in an organic solvent has proven to be efficient in producing individualized hydrophobic cellulose fibrils which can be readily and homogeneously dispersed in polyolefin matrices (Takase and Shiraishi 1989; Maldas and Kokta 1994; Bledzki et al. 1996; Gauthier et al. 1998; Sclavons et al. 2005; Qiu et al. 2006). The polyphenol tannic acid (TA) has also been demonstrated to functionalise the surface of MFC fibrils under alkaline conditions at room temperature (Lee et al. 2007; Ejima et al. 2013; Sileika et al. 2013). The hydrophilic product obtained can further react with primary amines (Lee et al. 2007; Ejima et al. 2013; Sileika et al. 2013; Hu et al. 2017). In this study the primary amines used are the short chain hexylamine $\left(\mathrm{C}_{6} \mathrm{H}_{13} \mathrm{NH}_{2}\right)$ and the hydrophobic long chain octadecylamine $\left(\mathrm{C}_{18} \mathrm{H}_{37} \mathrm{NH}_{2}\right)$, which form stable covalent bonds with the MFC-TA complex. The material obtained using the short chain hexylamine and the material obtained from the reaction with octadecylamine were filtered and oven dried. The resultant dry form is easy to grind at room temperature to obtain a powder. This can then be processed alongside polyolefins in a classical compounder to obtain MFC reinforced nanocomposites (see Supplementary Information, Figs. $1 \mathrm{~S}$ and $2 \mathrm{~S}$ ). The long aliphatic tail $\left(\mathrm{C}_{18}\right)$ of the octadecylamine renders the cellulose fibrils hydrophobic, and thereby supports the efficient dispersion of the reacted MFC in the composites. On the other hand the hexylamine short aliphatic tail $\left(\mathrm{C}_{6}\right)$ confers to the TA-MFC compound a less marked hydrophobic character, insufficient to obtain a good dispersion of the filler. This approach is favourable over other chemical treatment methods since it uses natural products for the modification of the cellulose, moving away from commonly used organic solvent approaches. 
In the present work a set of tannic acid-hexylamine treated MFC (MFC-TA- $\mathrm{C}_{6}$ ) reinforced composites are used as a comparison to tannic acid-octadecylamine treated MFC (MFC-TA-C 18 ) reinforced composites, and the dispersion of the reinforcing phase characterised using both ToF-SIMS and Spectral Confocal Laser Scanning Microscopy (SCLSM). The hypothesis is that this approach can better disperse the MFC within a hydrophobic resin, improving mechanical properties, and removing aggregates.

\section{Materials}

MFC slurry having a water content of $95 \mathrm{wt} \%$ was produced by FiberLean by the mechanical grinding of softwood bleached Kraft pine pulp. The poly(propylene)-poly(ethylene) compolymer (PPPE) matrix material (with a melting temperature of $170{ }^{\circ} \mathrm{C}$ ) was purchased from LyondellBasell (Rotterdam, Netherlands). Pure non-porous cellulose film was received from the Fraunhofer-Institut für Angewandte Polymerforschung (Geiselbergstr). The following chemicals and reagents were purchased from Sigma Aldrich (Dorset, UK): Tannic acid (TA) powder, octadecylamine $\left(\mathrm{C}_{18} \mathrm{H}_{37} \mathrm{NH}_{2}\right)$ powder technical grade $90 \%$, hexylamine $\left(\mathrm{C}_{6} \mathrm{H}_{13} \mathrm{NH}_{2}\right) 99 \%$, 4-(2-hydroxyethyl)-1piperazineethanesulfonic acid (HEPES) powder $>99.5 \%$, polypropylene-graft-maleic anhydride (MAgPP—average $\mathrm{Mw} \sim 9100$ and $\mathrm{Mn} \sim 3900$ by GPC), maleic anhydride 8-10 wt $\%$, acetone $\geq 99.9 \%$ $\left(\rho=0.79 \mathrm{~g} \mathrm{~cm}^{-3}\right)$ and anhydrous toluene, $99.8 \%$ $\left(\rho=0.87 \mathrm{~g} \mathrm{~cm}^{-3}\right)$. Xylene, 99\% $\left(\rho=0.88 \mathrm{~g} \mathrm{~cm}^{-3}\right)$ and sodium hydroxide reagent grade were supplied by Fisher Scientific (Leicestershire, UK).

\section{Experimental methods}

\section{Solvent swap}

$40 \mathrm{~g}$ of MFC slurry at $5 \mathrm{wt} \%$ of cellulose in water, containing $2 \mathrm{~g}$ of cellulose fibrils, was filtered and resuspended in $100 \mathrm{~mL}$ of acetone. The suspension was magnetically stirred for $10 \mathrm{~min}$ at $500 \mathrm{rpm}$ and then filtered under vacuum on a glass filter before resuspending in acetone; this procedure was repeated 3 times. The filtered material was then re-suspended in
$100 \mathrm{~mL}$ of toluene. The washing procedure was repeated 3 times with toluene. The solvent-exchanged material was filtered, recovered and further processed.

\section{MAgPP surface reaction}

The solvent-swapped, filtered MFC sample (2 $\mathrm{g}$ of MFC) was re-suspended in $100 \mathrm{~mL}$ of xylene and washed, as previously described, 2 times; the suspension was filtered and the filtercake was re-suspended in $160 \mathrm{~mL}$ of xylene in a round-bottomed, three-necked flask and heated up to $160{ }^{\circ} \mathrm{C}$. The suspension was kept at $160{ }^{\circ} \mathrm{C}$ (boiling point of xylene) and refluxed, under magnetic stirring at $500 \mathrm{rpm}$, for $10 \mathrm{~min}$ to eliminate residual water. $0.2 \mathrm{~g}$ of MAgPP was added through a separate funnel and the system was refluxed under magnetic stirring at $500 \mathrm{rpm}$ for $1 \mathrm{~h}$. The final solid product was filtered on a glass filter with a Venturi vacuum system, weighed and kept in a vacuum oven at $60{ }^{\circ} \mathrm{C}$ overnight.

Tannic acid-octadecylamine and tannic acidhexylamine surface reaction

$100 \mathrm{~g}$ of MFC slurry at $1 \mathrm{wt} \%$ of cellulose was diluted to a final volume of $500 \mathrm{~mL}$ with distilled water; $2.5 \mathrm{~g}$ of HEPES was added to the suspension and the $\mathrm{pH}$ was adjusted to 8 with sodium hydroxide. $0.5 \mathrm{~g}$ of tannic acid (TA) was added to the suspension and kept under magnetic agitation at $500 \mathrm{rpm}$ overnight at room temperature. $0.5 \mathrm{~g}$ of octadecylamine (melting point $50{ }^{\circ} \mathrm{C}$ ) was suspended in $50 \mathrm{~mL}$ of water at $70{ }^{\circ} \mathrm{C}$ by magnetic stirring the suspension at $500 \mathrm{rpm}$. The suspension was added to the MFC-TA reacted suspension and kept under magnetic stirring at $200 \mathrm{rpm}$ for $3 \mathrm{~h}$ at room temperature. The product was filtered on a paper filter (Whatman ${ }^{\mathrm{TM}} 541$-hardened ashless) under vacuum. The recovered material was resuspended in $100 \mathrm{~mL}$ of acetone, filtered under vacuum, recovered and kept in a fume cupboard to dry. The dried product was weighed and then passed through a laboratory grinder. The same procedure was followed to produce MFC samples functionalised with hexylamine in place of octadecylamine, replacing $0.5 \mathrm{~g}$ $(0.002 \mathrm{~mol})$ of octadecylamine with $0.2 \mathrm{~g}(0.002 \mathrm{~mol})$ of hexylamine. The same procedure used to prepare the octadecylamine reacted MFC was used to prepare hydrophobic non-porous cellulose films, substituting 
$100 \mathrm{~g}$ of MFC slurry at $1 \mathrm{wt} \%$ of cellulose with $1 \mathrm{~g}$ of non-porous cellulose film cut in strips of $1.5 \mathrm{~cm}$ width. After the reaction the strips were recovered, washed in an acetone bath and left to dry in a fume cupboard.

Preparation of nanocomposites

\section{Compounding}

Composites were prepared in a ZSK Mc ${ }^{18}$ counter rotating twin screw extruder (Coperion) with a specific torque of $18 \mathrm{Nm} \mathrm{cm}^{-3}$, maximum screw speed of $1200 \mathrm{~min}^{-1}$, and screw inner diameter ratio $\left(\mathrm{D}_{\mathrm{o}} / \mathrm{D}_{\mathrm{i}}\right)$ of 1.55 (8.2 mm screw diameter) using filler concentrations of $0.5,1,2,3,4,5,10,15$ and $20 \mathrm{wt} \%$. Specimens reinforced with $1 \mathrm{wt} \%$ polymerised TA were prepared using the powder obtained by the grinding of tannic acid powder (average particle diameter $\sim 30 \mu \mathrm{m}$ ) polymerised under alkali conditions overnight, to mimic the MFC-TA reaction. The samples were compounded at $210{ }^{\circ} \mathrm{C}$ at a $2 \mathrm{~kg} \mathrm{~h}^{-1}$ feed rate and $200 \mathrm{rpm}$. The obtained composite pellets were recovered for further processing.

\section{Preparation of specimens for testing}

Specimens for tensile and impact tests were injection moulded using an Arburg $221 \mathrm{M}$ machine, in accordance with ASTM D4761-13 and ASTM D 882. The specimens produced were Type I of ASTM D 882.

\section{Contact angle and free surface energy}

Contact angles of pure non-porous cellulose and TAoctadecylamine reacted non-porous cellulose films were measured using a Fibrodat 68-96 DAT Dynamic Absorption Tester (Testing Machines, Inc.). Sheets of untreated MFC, TA- $\mathrm{C}_{6}$ and TA- $\mathrm{C}_{18}$ treated MFC were obtained by the filtration of the MFC suspension using a standard handsheet former followed by pressing of the filtercake into a sheet and drying according to TAPPI procedure T205; untreated MFC sheets were also cut into strips for tensile modulus measurements. Contact angles on these were measured using the same instrument. De-ionized water was used as a probe liquid. The free surface energy of the pure and TAoctadecylamine reacted non-porous cellulose films and of MFC and TA-C $\mathrm{C}_{6}$ and $\mathrm{TA}-\mathrm{C}_{18}$ treated MFC sheets were evaluated using the Fibrodat tester with water as the polar probe liquid and bromonaphthalene $(\mathrm{BN})$ as the apolar probe liquid. The contact angle and free surface energy for the MFC films are reported in Supplementary Information. To minimize experimental errors, the values were measured at five random locations for each sample, and an average reported.

Energy-dispersive X-ray spectroscopy (EDX) imaging

A JEOL IT300 Scanning Electron Microscopy (SEM) operated at $2 \mathrm{keV}$ and a working distance of $15 \mathrm{~mm}$ was used to image $\mathrm{TA}-\mathrm{C}_{18}$ treated MFC reinforced composites. The samples were carbon coated and analysed using Energy-Dispersive X-ray spectroscopy (EDX) imaging.

\section{Time of Flight Secondary Ion Mass Spectroscopy} (ToF-SIMS) mapping

ToF-SIMS images were acquired at Nottingham University using a ToF-SIMS IV instrument (IONTOF GmbH, Münster, Germany) equipped with a bismuth liquid metal ion gun and a single-stage reflectron analyser. Operating conditions utilized $\mathrm{Bi}^{3+}$ ions with a primary energy of $25 \mathrm{kV}$ and a pulsed target current of approximately $1.0 \mathrm{pA}$. Lowenergy electrons $(20 \mathrm{eV})$ were used to compensate for surface charging caused by the positively charged primary ion beam on the insulating surfaces. Individual spots were analysed by rastering areas of $500 \times 500 \mu \mathrm{m}$ at a resolution of $256 \times 256$ pixels. The total primary ion beam dose for each analysed area was kept below $1 \times 10^{12}$ ions $\mathrm{cm}^{-2}$, ensuring static conditions.

\section{Multi-channel spectral confocal laser scanning microscopy (SCLSM) mapping}

Composite samples were cryo-microtomed into slices of $20 \mu \mathrm{m}$ thickness cut from the central part of a dumbbell having dimensions of $10 \times 4 \mathrm{~mm}$ (length $\times$ width). Slices were placed between a glass slide and a coverslip to flatten the surface. Spectral $z$ stack images $(800 \times 800 \mu \mathrm{m})$ were generated using a Zeiss LSM 880 confocal microscope $(405 \mathrm{~nm}$ diode laser, $5.0 \%$ power, Plan-Apochromat $10 \times / 0.45$ M27 objective, MBS-405 filter, 32 channels: 
$\lambda=411-695 \mathrm{~nm})$. The maximum distance between stack slices was $\sim 2 \mu \mathrm{m}$.

\section{Image processing}

Image stacks generated using spectral confocal microscopy were processed in Fiji software. The zprojection function (projection type: standard deviation) was used to flatten image stacks into single images. After thresholding, images were analysed to determine the observed aggregate areas. Aggregates at the edge of the images were excluded, as were aggregates smaller than $11 \mu^{2}$, i.e. less than four pixels, as these could not be visually identified.

\section{Aggregate analysis}

Aggregate distribution was subdivided into four categories: small, medium, large and outliers. Rather than set these categories between fixed area values, the maximum and minimum values for each category were determined using the box plots themselves. A box plot was constructed using the entire data set, and the values at which data would be classified as an upper, or lower, outlier determined. The box plot was then regenerated using the outlier values as the maximum and minimum for the data range and new outlier values calculated. This process was repeated until the range of values fell between the upper and lower outlier values. This determined the aggregates that fell into the small category for each sample. To determine the medium category range, the process was repeated excluding all values in the small category. The process was repeated excluding values in the small and medium categories to define the large category range. All values that fell out of these ranges were classified as outliers. Due to the skew present in the data sets, the calculated lower outlier values were always less than the initial lower data values for all samples. To compare the aggregate populations, the boundaries for the four size categories were set as the boundaries determined for the $1 \mathrm{wt} \%$ MFC-TA- $\mathrm{C}_{18}$ composite sample using the above technique.

\section{Statistical analysis}

For the comparison of the composite mean aggregate areas and aggregate population analysis, a one-way analysis of variance (ANOVA) test was used to determine the statistical differences between two or more samples, assuming equal variance, with Tukey HSD posthoc correction. A confidence interval of 0.95 was used.

\section{Mechanical and impact tests}

Mechanical tests were conducted using a tensile testing machine (Instron 3367). The maximum load and the crosshead speed used were $30 \mathrm{kN}$ and $10 \mathrm{~mm} \mathrm{~min}^{-1}$. The tests were performed in accordance with ASTM D4761-13 and ASTM D 882. The impact tests were conducted using an impact testing machine (Instron-CEAST 9340-Drop Tower Impact System) at room temperature. The tests were performed according to ISO-179 2.

\section{Results and discussion}

Contact angle and free surface energy

The contact angles and the free surface energies of the pure matrix (PPPE), untreated and TA- $\mathrm{C}_{18}$ reacted non-porous cellulose films are provided in Table 1. The values of contact angle and free surface energy for porous MFC films are reported in Table 1S in Supplementary Information. The values were calculated from five different sampling points on the same specimen to minimize the errors. The free surface energy was evaluated using water and bromonaphthalene (BN) and applying the Owens, Wendt, Rabel and Kaelble method according to the equation

$\sigma_{\mathrm{sl}}=\sigma_{\mathrm{s}}+\sigma_{\mathrm{l}}-2\left[\left(\sigma_{\mathrm{sw}} \sigma_{\mathrm{lw}}\right)^{\frac{1}{2}}+\left(\sigma_{\mathrm{sd}} \sigma_{\mathrm{ld}}\right)^{\frac{1}{2}}\right]$.

combined with Young's equation

$\sigma_{\mathrm{s}}=\sigma_{\mathrm{sl}}+\sigma_{1} \cos \theta$

where $\sigma_{\mathrm{s} 1}$ is the solid-liquid interfacial tension, $\sigma_{\mathrm{s}}$ is the solid-air surface tension and $\sigma_{1}$ is the liquid-air surface tension, $\sigma_{\mathrm{sw}}$ is the polar component of the solid-air surface tension, $\sigma_{\mathrm{lw}}$ is the polar component of the liquid-air surface tension, $\sigma_{\mathrm{sd}}$ is the dispersed component of the solid-air surface tension, $\sigma_{\mathrm{ld}}$ is the dispersed component of the liquid-air surface tension, and $\theta$ is the measured contact angle. The Owens, Wendt, Rabel and Kaelble method relies on the data collected for at least two liquids, one polar and one 
Table 1 Free surface energy, polar and apolar contribution, water and BN contact angle of PPPE, untreated non-porous cellulose, and $\mathrm{TA}-\mathrm{C}_{18}$ reacted non-porous cellulose films

\begin{tabular}{|c|c|c|c|c|c|}
\hline & \multirow{2}{*}{$\begin{array}{l}\text { Free surface energy } \\
\left(\mathrm{mN} \mathrm{m}^{-1}\right)\end{array}$} & \multirow{2}{*}{$\begin{array}{l}\text { Polar contribution } \\
\left(\mathrm{mN} \mathrm{m}^{-1}\right)\end{array}$} & \multirow{2}{*}{$\begin{array}{l}\text { Dispersive contribution } \\
\left(\mathrm{mN} \mathrm{m}^{-1}\right)\end{array}$} & \multicolumn{2}{|c|}{ Contact angle $\left(^{\circ}\right)$} \\
\hline & & & & Water & $\mathrm{BN}$ \\
\hline PPPE & $37.6 \pm 0.2$ & $3.6 \pm 0.1$ & $33.9 \pm 0.1$ & $83 \pm 1$ & $41 \pm 1$ \\
\hline Non-porous cellulose & $70.1 \pm 0.1$ & $36.1 \pm 0.1$ & $34.6 \pm 0.1$ & $21 \pm 1$ & $45.1 \pm 0.7$ \\
\hline $\begin{array}{l}\text { Non-porous cellulose } \\
\text { TAC }_{18}\end{array}$ & $33.9 \pm 0.1$ & $1.2 \pm 0.1$ & $32.8 \pm 0.1$ & $93 \pm 1$ & $44 \pm 0.5$ \\
\hline
\end{tabular}

apolar. From the two sets of contact angle data it is possible to write a system of two equations which is solved by the geometrical mean method giving a value for the solid-air surface tension $\left(\sigma_{\mathrm{s}}\right)$

The TA- $\mathrm{C}_{18}$ surface treatment of the non-porous cellulose film increases the sample water contact angle from $21^{\circ}$ to $93^{\circ}$ and decreases the free surface energy of the samples from 70.1 to $33.9 \mathrm{mN} \mathrm{m}^{-1}$ respectively. The non-porous cellulose films were used as a model system because the porous nature of MFC films made it impossible to obtain reliable values for the contact angle and free surface energy. Nevertheless, the measured water contact angle of MFC varies from $44^{\circ}$ for the untreated sample to $121^{\circ}$ for the $\mathrm{TA}-\mathrm{C}_{18}$ surface reacted MFC film.

The contact angle data for both non-porous cellulose and MFC shows that the surface modification of cellulose by the reaction with TA and octadecylamine decreases the surface energy of the cellulose substantially, and thus should improve its dispersibility in apolar polymers. The contact angle of MAgPP reacted MFC was not measured as it was impossible to form a coherent sheet. The time evolution of the polar and apolar contact angle with water and $\mathrm{BN}$ probe on the different supports (Fig. 3S and 4S) can be found in Supplementary Information.

\section{EDX imaging of MFC reinforced composites}

The composites obtained by reinforcing the neat matrix with $1 \mathrm{wt} \%$ MAgPP-MFC and $0.5 \mathrm{wt} \%$, $1 \mathrm{wt} \%$ and $15 \mathrm{wt} \%$ MFC-TA-C ${ }_{18}$ were investigated using EDX imaging. EDX analysis of the transverse surface of the composites was used to investigate the filler dispersion. MAgPP reacted MFC was used as a reference to determine the level of dispersion using a solvent based cellulose surface chemistry.

A typical aggregate of MAgPP-MFC is reported in Fig. 1; the dimension of this aggregate was evaluated to be $\sim 100 \mu \mathrm{m}$ in length. Similar aggregates were found within the MFC-TA- $\mathrm{C}_{18}$ reinforced samples (Fig. 2).

EDX spectroscopic evidence suggests that there is an incomplete dispersion of the MFC, resulting in the formation of aggregates. Aggregates in samples containing MAgPP treated MFC are typically different sizes from those in samples containing $\mathrm{TA}-\mathrm{C}_{18}$ treated MFC. The presence of small aggregates $(10 \mu \mathrm{m}$ or below) and a semi-continuous background is noted. This latter result suggests that a fraction of the filler can be dispersed in the matrix at a much smaller scale compared to the size of the large visible aggregates. Unfortunately, EDX cannot fully discriminate the signal from cellulose since the presence of the oxygen from the background could be related to contamination of the specimens.

\section{ToF-SIMS chemical mapping}

It is difficult to identify cellulose unequivocally using EDX, which is also a demanding technique in terms of sample preparation. For those reasons, ToF-SIMS and SCLSM were also used to evaluate the dispersion of the filler. ToF-SIMS analysis was used to produce 2D maps of the distribution of cellulose within the composites. In the analysis, characteristic secondary ion peaks were assigned to the matrix (fragment $\mathrm{C}_{5} \mathrm{H}_{9}{ }^{+}$at $69.1 \mathrm{~m} / \mathrm{z}$ ) and to the cellulose (fragment $\mathrm{C}_{14} \mathrm{H}_{23} \mathrm{O}_{5}{ }^{+}$at $270.3 \mathrm{~m} / \mathrm{z}$ ). Figures 3 and 4 indicate the relative $\mathrm{m} / \mathrm{z}$ and normalised ion intensities of the characteristic peaks selected as references. Aggregates 

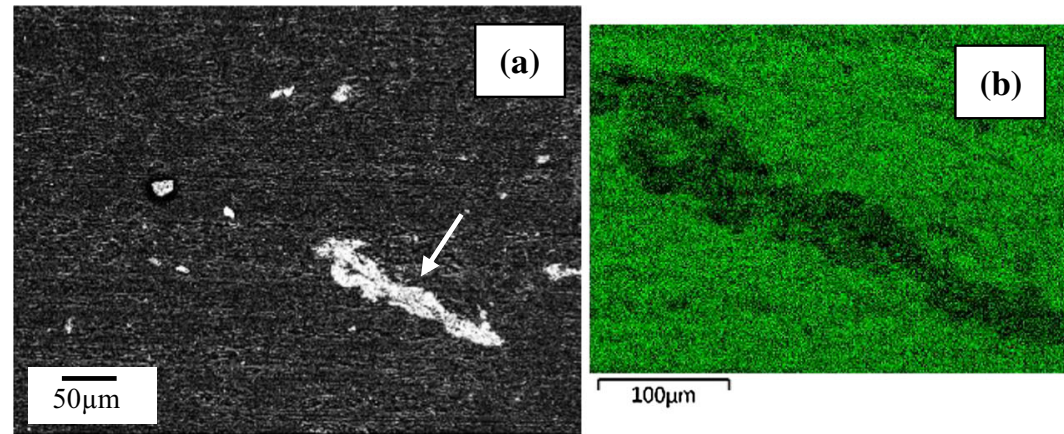

$100 \mu \mathrm{m}$

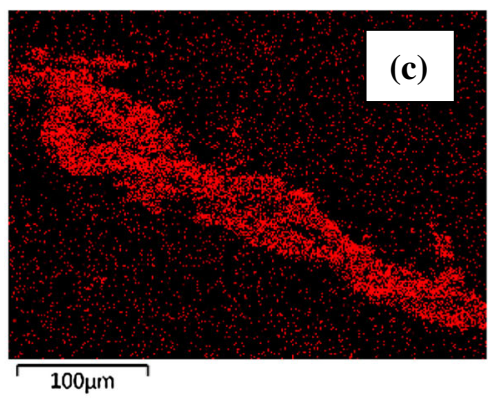

arrow); b back-scattered image of the large agglomerate where carbon is identified with a green colour; $\mathbf{c}$ back-scattered image of the agglomerate, where oxygen is identified in red
Fig. 1 Typical Back-scattered Electron EBSD and energ dispersive X-ray spectroscopy (EDX) images of $1 \mathrm{wt} \%$ MAgPP-MFC reinforced composites. From left to right: a grayscale image of a large agglomerate (identified with an
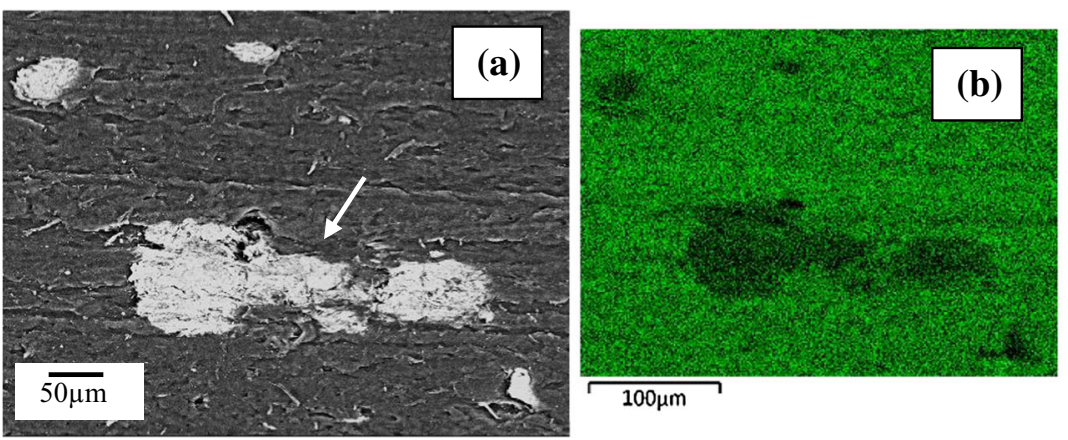

$100 \mu \mathrm{m}$

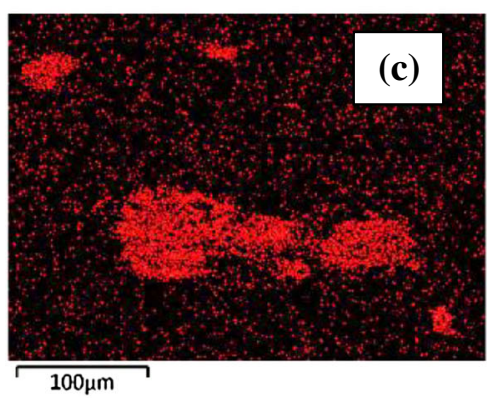

Fig. 2 Typical Back-scattered Electron EBSD and energydispersive X-ray spectroscopy (EDX) images of 1\% MFC-TA$\mathrm{C}_{18}$ reinforced composites. From left to right: a grayscale image of a large agglomerate (identified with an arrow); b back-

in the composites containing $5 \mathrm{wt} \%$ of MFC-TA- $\mathrm{C}_{6}$ were very large and easy to identify, and so were assumed to be comprised mostly of cellulose and used to verify the peak assignment (Fig. 5). Hexylamine is a water-soluble amine which should react with tannic acid in the same way as octadecylamine; however, its chain length appears insufficient to disperse the cellulose in the polymer matrix. Large MFC aggregates can been seen in the composites (Fig. 1S). In the $2 \mathrm{D}$ reconstruction of the reference system, the cellulose signal located at $270 \mathrm{~m} / \mathrm{z}$, identified by a blue colour, is very distinctive from the signal from the matrix (in red; Fig. 5b). This shows that the ToF-SIMS mapping has successfully resolved the cellulose filler from the matrix. ToF-SIMS $2 \mathrm{D}$ analysis was used to obtain the map for a $5 \mathrm{wt} \%$ MFC-TA- $\mathrm{C}_{18}$ reinforced composites sample; the area selected did not exhibit scattered image of the large agglomerate where carbon is identified with a green colour; $\mathbf{c}$ back-scattered image of the agglomerate, where oxygen is identified in red

aggregates of large dimensions (Fig. 6a). The 2D reconstructed map (Fig. 6b) indicated a more homogeneous dispersion of the hydrophobic filler.

\section{SCLSM spectra}

Previous research has confirmed that SCLSM can be used to identify cellulose aggregates in composite materials without the need for a fluorescent dye (Johns et al. 2019). Here we confirmed the autofluorescence of microfibrillated cellulose (MFC), tannic acid (TA) and the MFC-TA- $\mathrm{C}_{18}$ filler in composites. This ensured that the CLSM images of the composite samples enabled identification of the aggregates (Fig. 7), for all composites. Aggregates observed in the $1 \mathrm{wt} \%$ MFC and $1 \mathrm{wt} \%$ MFC-TA- $\mathrm{C}_{18}$ composites had similar spectra, whilst the $1 \mathrm{wt} \%$ TA aggregates 


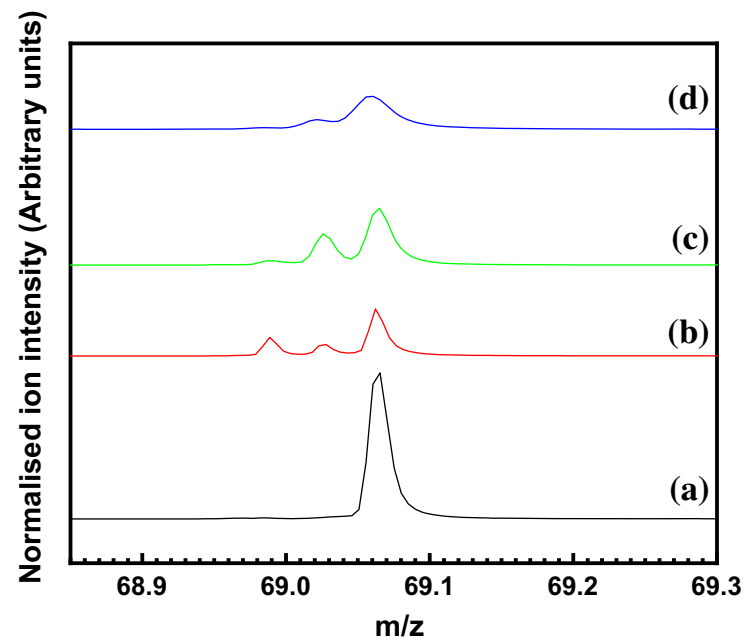

Fig. 3 Typical ToF-SIMS mass spectra magnification of peaks corresponding to the polymer matrix (fragment $\mathrm{C}_{5} \mathrm{H}_{9}{ }^{+}$at $69.1 \mathrm{~m} / \mathrm{z})$; (a), pure tannic acid (b), untreated MFC (c), and MFC-TA-C ${ }_{18}(\mathbf{d})$ samples

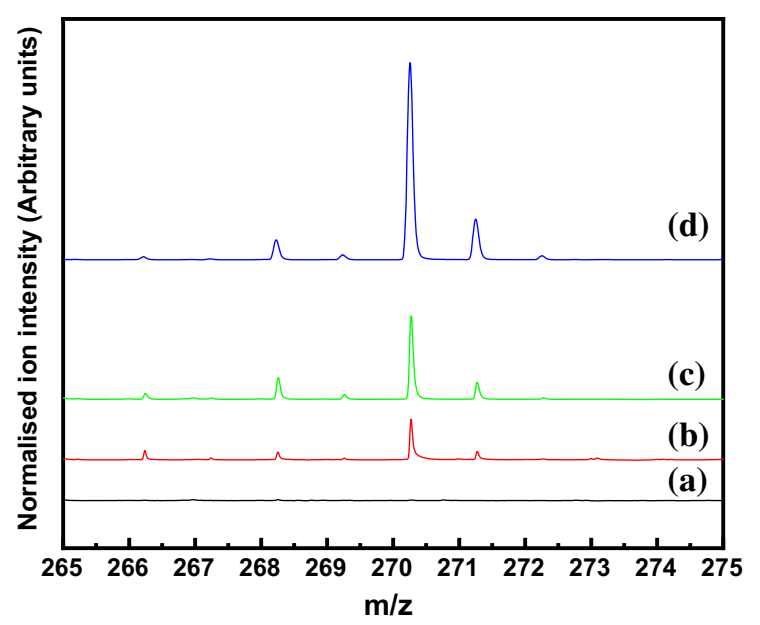

Fig. 4 ToF-SIMS mass spectra magnification of the peaks corresponding to cellulose (fragment $\mathrm{C}_{14} \mathrm{H}_{23} \mathrm{O}_{5}{ }^{+}$at $270.3 \mathrm{~m} / \mathrm{z}$ ); (a), pure tannic acid (b), untreated MFC (c), and MFC-TA-C 18 (d) samples

were less intense (Fig. 8a), in line with spectra from the raw materials (Fig. 8b).

The background spectra for the composites all contain a clearly defined matrix peak and a contribution from the composite material (Fig. 8b). The $1 \mathrm{wt} \%$ MFC-TA- $\mathrm{C}_{18}$ sample background appears to have a honeycomb-like structure (Fig. 7c and Fig. 5S). The origin of this structure is unclear and requires further investigation. It is presumed that the $15 \mathrm{wt} \% \mathrm{MFC}$ -
TA- $\mathrm{C}_{18}$ composite has a similar structure, but the increase in intensity obscures this. In addition, this sample (Fig. 7d) exhibits a uniform background fluorescence, which we assume is the dispersion of non-aggregated cellulose, although we do not have definitive evidence for this.

\section{Aggregate size and distribution obtained by SCLSM}

The modification of MFC with $\mathrm{TA}-\mathrm{C}_{18}$ results in a significant increase in the number of aggregates observed, and a decrease in the mean area of the observed aggregates compared with those in the $1 \mathrm{wt} \%$ MFC and $1 \mathrm{wt} \%$ TA composites (Fig. 9). This suggests that the modification improves the distribution of material throughout the polymer composite. As expected, an increase in the MFC-TA- $\mathrm{C}_{18}$ loading results in an increase in the number of aggregates observed but has no effect on the mean aggregate area observed.

Division of the aggregate areas into small, medium and large categories confirms the differences in distribution between composite samples (Fig. 10). Whilst the $1 \mathrm{wt} \%$ and $15 \mathrm{wt} \%$ MFC-TA-C 18 samples are similar, the $1 \mathrm{wt} \%$ MFC aggregates are much larger; for example, both the median and mean values for the large aggregates are an order of magnitude greater than those for the MFC-TA- $\mathrm{C}_{18}$ samples. The fact that the $1 \mathrm{wt} \%$ TA, $1 \mathrm{wt} \%$ MFC-TA-C 18 and $15 \mathrm{wt} \%$ MFC-TA- $\mathrm{C}_{18}$ samples have no lower bound for the small category suggests that there are aggregates present that are smaller than the minimum viewable area $\left(\sim 11 \mu \mathrm{m}^{2}\right)$. It is also worth noting that, whilst there appear to be many outlier values for the $15 \mathrm{wt} \%$ MFC-TA-C ${ }_{18}$ sample, they consist of less than $1 \%$ of the total number of aggregates observed, and they fall within the same range as the MFC aggregates.

By setting the $1 \mathrm{wt} \%$ MFC-TA- $\mathrm{C}_{18}$ composite as the control for defining the aggregate area categories, it is possible to compare the population distribution across the samples (Fig. 11). Under these boundary conditions, the distribution of aggregates for the $1 \mathrm{wt} \%$ MFC composite is quite even; $31 \pm 4 \%$ of aggregates fall into the small category, whilst $17 \pm 5 \%$ are classified as outliers. The TA aggregates are significantly skewed towards the small category; $61 \%$ of aggregates fall into this category. There is, 


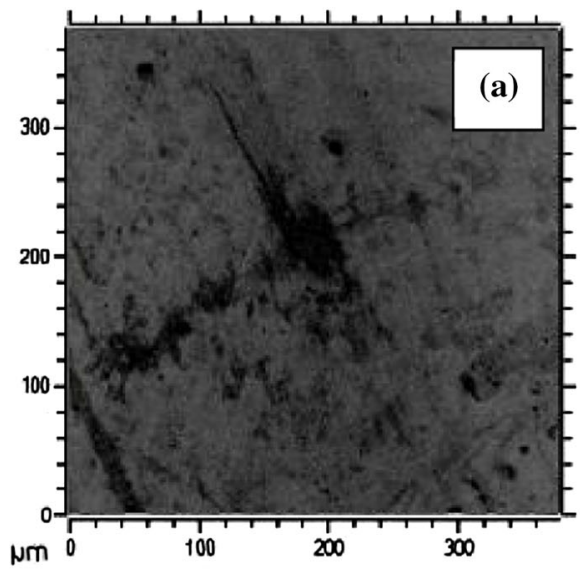

Fig. 5 Typical ToF-SIMS images of the MFC-TA- $\mathrm{C}_{6}$ reinforced composite used verify the peak assignment. a Optical image of the sample (grayscale), with a large agglomerate of cellulose reinforcement visible in the centre of the sample and

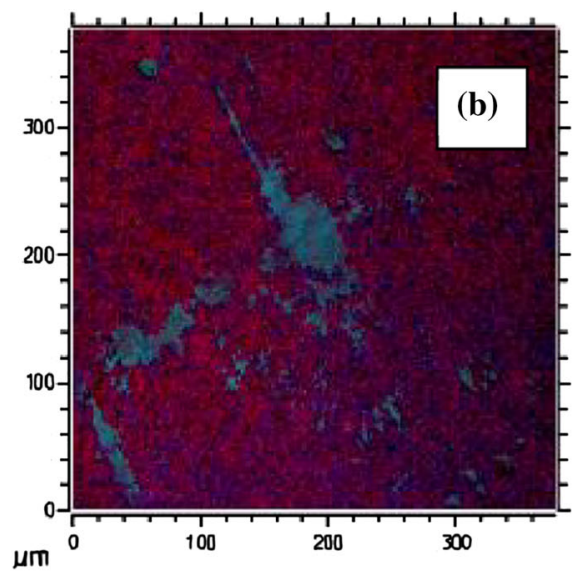

b a $2 \mathrm{D}$ reconstruction of the composite system using the assigned peaks: the red colour indicates the matrix and the blue colour indicates the presence of cellulose
Fig. 6 Typical ToF-SIMS images of the MFC-TA- $\mathrm{C}_{18}$ reinforced composite. The grayscale image a represents the optical image of the sample-no large cellulose agglomerates are visible in the sample; $\mathbf{b}$ is a $2 \mathrm{D}$ reconstruction of the composite system: the red colour indicates the matrix and the blue colour indicates the presence of cellulose
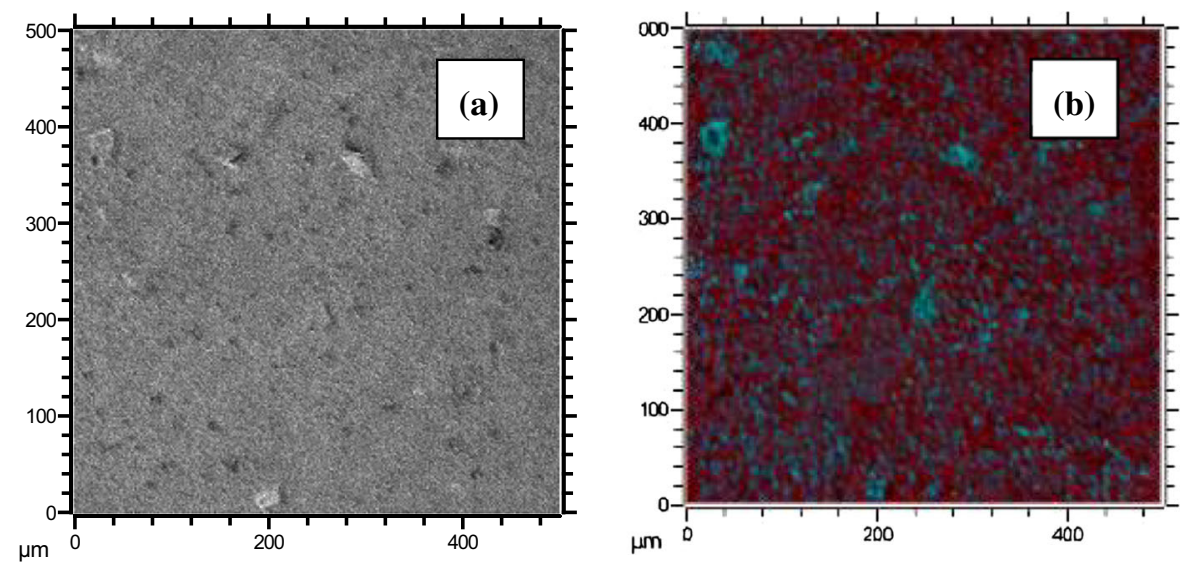

however, no significant difference between the MFC and TA aggregates for the other three categories. This analysis also confirms that the modification of MFC with TA-C ${ }_{18}$ significantly improves the distribution of the material within the matrix. Over $85 \%$ of the aggregates fall into the small category for both 1 and 15 wt $\%$ MFC-TA- $\mathrm{C}_{18}$ composites, with less than $3 \%$ of the aggregates falling into the large and outlier categories combined. This results in composites that are significantly different to the $1 \mathrm{wt} \%$ MFC sample across all categories and are also significantly different to the $1 \mathrm{wt} \%$ TA composite in the small and medium categories. Importantly, no significant difference is observed in the population distribution between the
$1 \mathrm{wt} \%$ and $15 \mathrm{wt} \%$ MFC-TA- $\mathrm{C}_{18}$ composites, indicating that the increased loading does not result in aggregation of the MFC-TA- $\mathrm{C}_{18}$ particles.

\section{Mechanical and impact properties of composites}

The mechanical properties of pure PPPE matrix and MFC-TA- $\mathrm{C}_{18}$ reinforced composites were obtained from the average of five tested specimens. Young's modulus was calculated from the slope of the stressstrain curve between 0 and $0.2 \%$ strain.

Figure 12 presents tensile and impact properties of the neat matrix and composites reinforced with $0.5 \mathrm{wt} \%$ up to $15 \mathrm{wt} \%$ of MFC-TA- $\mathrm{C}_{18}$. The addition 


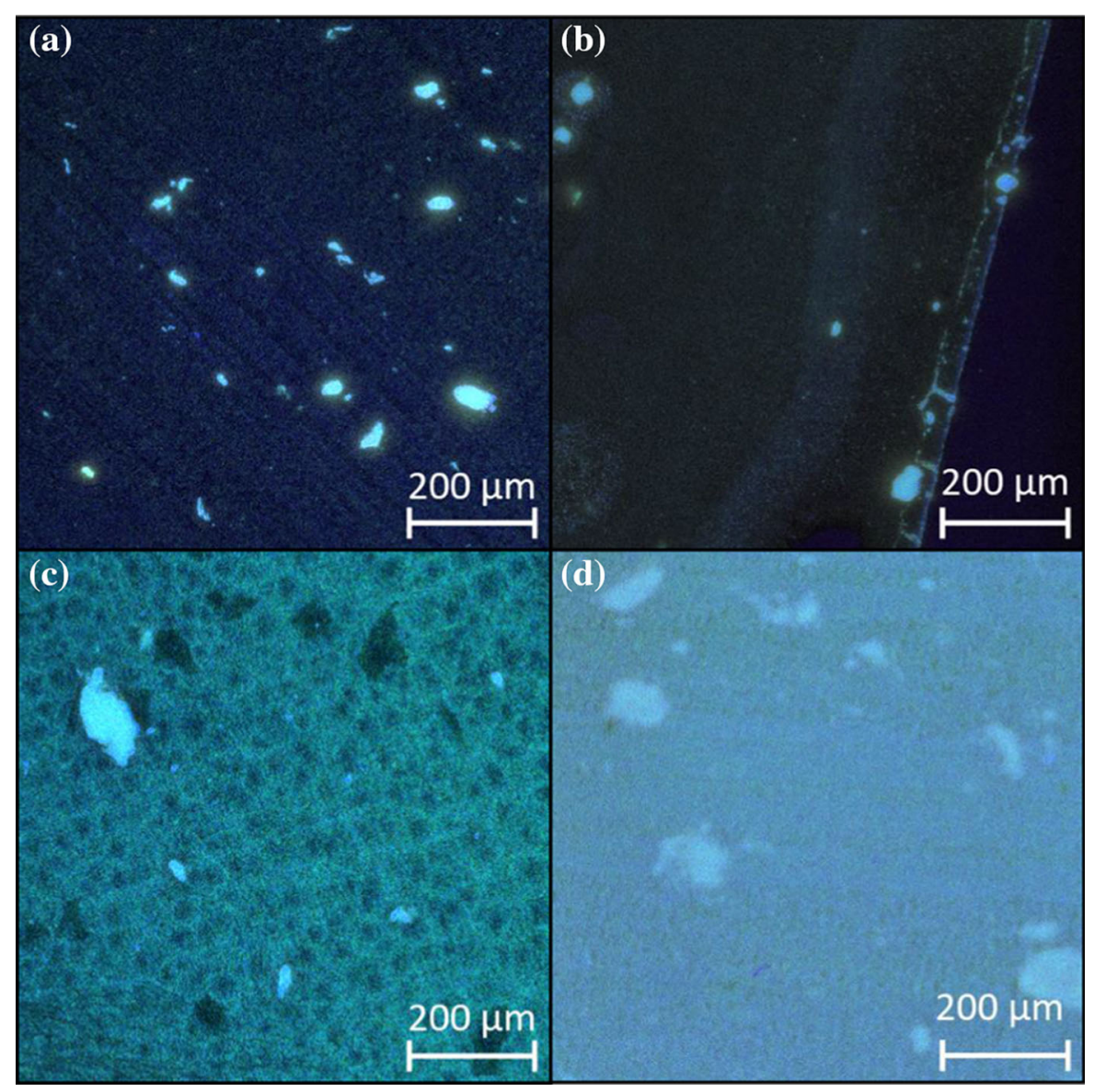

Fig. 7 Typical SCLSM images of a 1 wt $\%$ MFC, b 1 wt $\%$ TA, the visible fibre-like structure is a cutting mark, $\mathbf{c} 1 \mathrm{wt} \%$ MFCTA- $\mathrm{C}_{18}$, and d $15 \mathrm{wt} \%$ MFC-TA- $\mathrm{C}_{18}$ composites. The images

of reinforcement up to $2 \mathrm{wt} \%$ did not significantly enhance the tensile modulus of the composites. This increase in the reinforcing phase did however have an impact on the fracture energy of the specimens. Further increase in the filler concentration results in enhanced tensile properties but in a rapid decrease in the impact properties of the composites. Fracture energy increases from $\sim 76 \mathrm{~kJ} \mathrm{~m}^{-2}$ (pure PPPE) to $\sim 101 \mathrm{~kJ} \mathrm{~m}^{-2}$ at $1 \% \mathrm{MFC}$ content (see Fig. 12 and Supplementary Information, Table $2 \mathrm{~S}$ ). This then progressively decreases at higher fractions of MFC, which is thought to be due to the presence of aggregates in the sample.

Large filler aggregates were found in the specimens with low and high filler contents, as demonstrated by SCLSM. The analysis of the aggregate size and distribution indicated that, whilst the aggregate size are flattened from 3D stacks using the standard deviation z-project function in Fiji software

is not dependent on the filler content, the number of aggregates found in the reinforced composites increases with it. Most aggregates had a viewable area between 11 and $30 \mu \mathrm{m}^{2}$ and were classified as small particles. The filler aggregation in PPPE composites is thought to affect stiffness only marginally, but to strongly influence the impact resistance, which decreases sharply as aggregation increases (Fekete et al. 1999). In nanocomposites the reinforcement carries the tensile load while the matrix transfers this load between the reinforcement particles (Jager and Fratzl 2000). To ensure the integrity of the composite structure, the reinforcement should be able to withstand large tensile stress without fracture, whilst the matrix should carry a large shear stress without failure. The fracture resistance of a brittle solid is influenced by the flaw size according to the Griffith criterion 


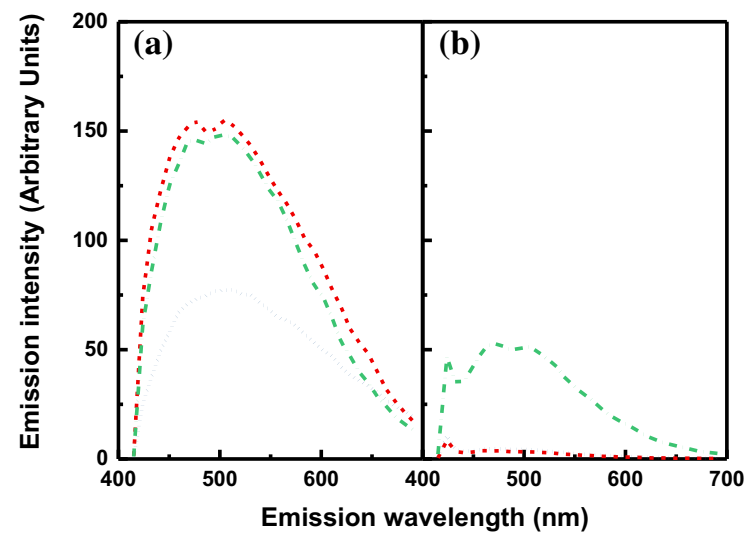

Fig. 8 Typical emission spectra of $\mathbf{a}$ aggregates and $\mathbf{b}$ the background for $1 \mathrm{wt} \%$ MFC (dashed red line), $1 \mathrm{wt} \%$ TA (dotted blue line), and $1 \mathrm{wt} \%$ MFC-TA- $\mathrm{C}_{18}$ (dash-dot green line). Note that the aggregates for $1 \mathrm{wt} \% \mathrm{MFC}$ and $1 \mathrm{wt} \% \mathrm{MFC}-$ TA- $\mathrm{C}_{18}$ have similar intensities, whilst $1 \mathrm{wt} \% \mathrm{TA}$ is less intense. Note also that the $1 \mathrm{wt} \%$ MFC-TA- $\mathrm{C}_{18}$ background spectrum is more intense than the other two spectra and consists of signals from both the PPPE matrix and MFC-TA- $\mathrm{C}_{18}$

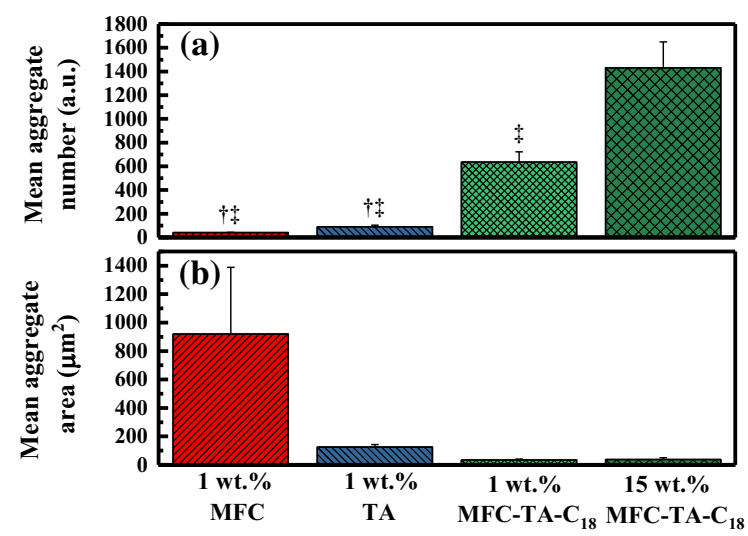

Fig. 9 a Mean number of aggregates for each composite sample. $\mathrm{N}=3$. $\uparrow p<0.05$ compared to $1 \mathrm{wt} \%$ MFC-TA-C $18 ; \dagger$ $p<0.05$ compared to $15 \mathrm{wt} \%$ MFC-TA-C 18 . Error $\pm \mathrm{SE}$; b mean aggregate area for each composite sample. No statistical difference was observed between samples. $\mathrm{N}=3, \mathrm{n} \geq 35$. Error \pm SE. a.u. $=$ arbitrary units

$\sigma_{r}^{f}=\alpha E_{r} \Psi$

$\Psi=\sqrt{\frac{\gamma}{E_{r} h}}$

where $\sigma_{r}^{f}$ is fracture strength of the material (in this case an aggregate of cellulose in a matrix), $E_{r}$ is the theoretical modulus of the reinforcement, in this case a cellulose fibril, $\gamma$ is the interfacial surface energy

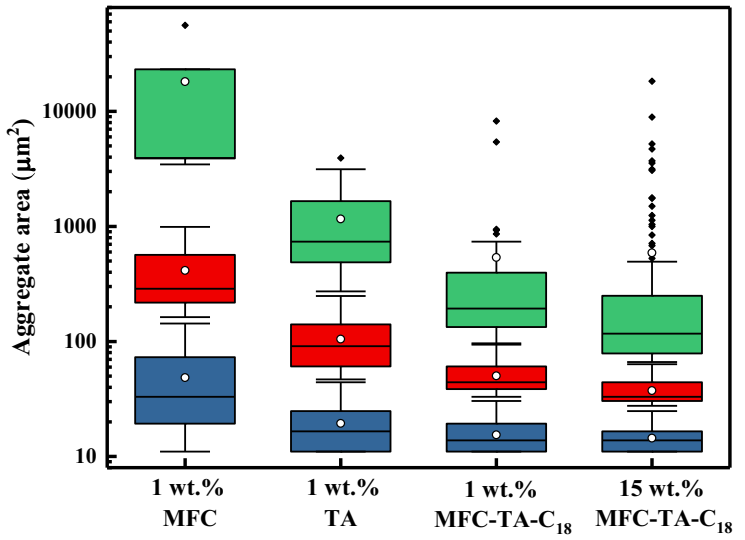

Fig. 10 Box plot comparing distribution of aggregates between samples obtained using spectral confocal microscopy. Aggregates are divided into four categories: small (blue boxes), medium (red boxes with light spot scattering), large (green boxes with medium spot scattering), and outliers (black diamonds). The mean values for each category are represented by white circles. Aggregates $<11 \mu \mathrm{m}^{2}$ ignored for analysis. $\mathrm{n}>120$

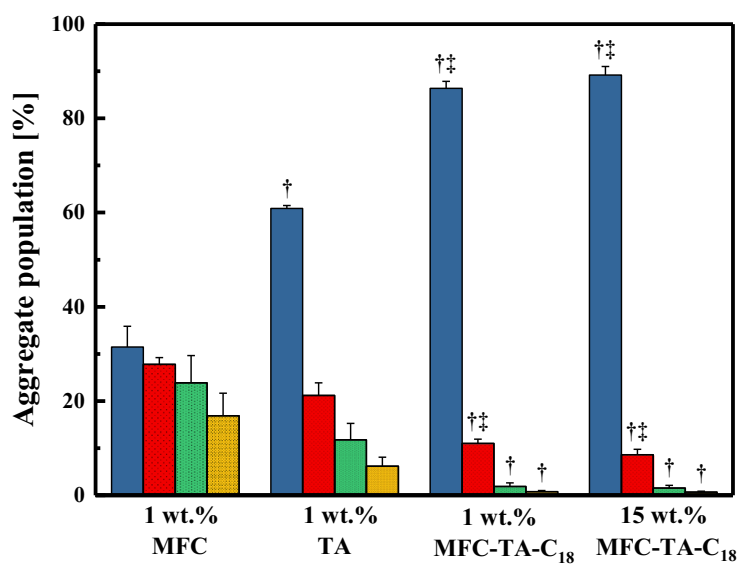

Fig. 11 Aggregate populations for various composites obtained via SCLSM. Aggregate categories, as defined by ranges determined for $1 \mathrm{wt} \%$ MFC-TA- $\mathrm{C}_{18}$ : small (blue bars, $11.0 \geq X \leq 30.3 \mu \mathrm{m}^{2}$ ), medium (red bars with light spot scattering, $30.3>X \leq 93.8 \mu \mathrm{m}^{2}$ ), large (green bars with medium spot scattering, $93.8>\mathrm{X} \leq 385.3 \mu^{2}$ ), and outliers (yellow bars with heavy spot scattering, $\mathrm{X}>385.3 \mu \mathrm{m}^{2}$ ). For all categories $\mathrm{N}=3 . \dagger p<0.05$ compared to $1 \mathrm{wt} \% \mathrm{MFC}$ in the respective category; $₫ p<0.05$ compared to $1 \mathrm{wt} \%$ TA in the respective category. Error $\pm \mathrm{SE}$

between the reinforcement and the matrix, and $h$ is the thickness of the reinforcement. The parameter $\alpha$ depends on the crack geometry and can be considered approximately equal to $\sqrt{\pi}$. Below a defined reinforcement thickness $\left(h^{*}\right)$ the fracture strength of a 


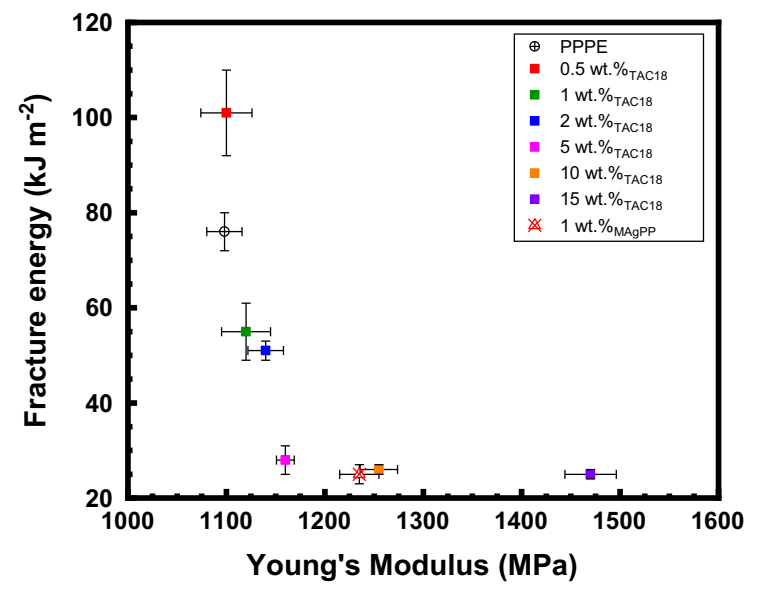

Fig. 12 Young's modulus and fracture energy of pure matrix (PPPE), $1 \mathrm{wt} \%$ MFC-MAgPP reinforced composites, and MFCTA- $\mathrm{C}_{18}$ reinforced composites at filler loadings from 0.5 up to $15 \mathrm{wt} \%$. These data, along with tensile strength (at yield and break), fracture energy and strain to failure, are available in Table $2 \mathrm{~S}$ in the supplementary information section

cracked reinforcement particle becomes identical to that of a flawless structure (Jager and Fratzl 2000). It is possible to estimate the critical aggregate length scale as

$h^{*} \approx \alpha^{2} \frac{\gamma E_{r}}{\sigma_{t h}^{2}}$

The values considered for the calculation are $\gamma=0.0339 \mathrm{~J} / \mathrm{m}^{2}$ calculated for the tannic acid-octadecylamine reacted MFC film, $E_{r}=20 \mathrm{GPa}$ and $\sigma_{t h}$ represents the theoretical tensile strength of cellulose fibrils and is equal to $240 \mathrm{MPa}$ (Zimmermann et al. 2004, 2005; Leitner et al. 2007). Using these values we estimate $h^{*}$ to be $\approx 37 \mathrm{~nm}$. The dimension of the small aggregate found using the SCLSM analysis accounts for $11 \mu \mathrm{m}^{2}$ and thus a length of about $2 \mu \mathrm{m}$, assuming a circular geometry to the aggregates. The small aggregates found in the composites exceed the critical aggregate length scale $(0.037 \mu \mathrm{m})$ by several orders of magnitude. This indicates that the cellulose filler is not sufficiently dispersed to obtain its full potential as a reinforcement without compromising fracture toughness.

The presence of aggregates also reduces the area of the filler matrix interface, limiting the effectiveness of MFC as reinforcement. Using the modulus of the composite as the measure, the potential of MFC as a reinforcement for the PPPE matrix was estimated using the 'Rule of Mixtures', which is expressed by the equation

$E_{\text {composite }}=\eta_{0} \eta_{1} E_{\text {fibril }} V_{\text {fibril }}+\left(1-V_{\text {fibril }}\right) E_{\text {matrix }}$

where $E_{\text {composite }}$ is the modulus of the composite, $V_{\text {fibril }}$ is the volume fraction of the fibres (or fibrils) in the composite and $E_{\text {matrix }}$ is the modulus of the matrix; $\eta_{1}$ and $\eta_{0}$ are the fibre length and orientation efficiency factors: $\eta_{1}$ is equal to 1 for long fibres, and $\eta_{0}$ is equal to $3 / 8$ for an in-plane random orientated network. The mechanical properties of a single cellulose fibril were evaluated using the Cox equation (Cox 1952) for an in plane random network of fibres, using an experiment in which sheets of pure MFC were made, cut into strips, and their tensile moduli were measured. The tensile modulus of the sheets, $E_{\text {network }}$, was found to be approximately $3.5 \mathrm{GPa}$. The modulus for a single cellulose fibril can be calculated using the equation

$\mathrm{E}_{\text {fibril }}=\frac{8}{3} \times \mathrm{E}_{\text {network }}$

This gave an experimental value of $\mathrm{E}_{\mathrm{fibril}}$ of $10 \mathrm{GPa}$. By comparison, the extrapolation of the moduli of the composites to $100 \%$ filler content, using the fit obtained in Fig. 13, gives an $\mathrm{E}_{\text {network }}$ modulus of $3.4 \mathrm{GPa}$, resulting in an effective value of $\mathrm{E}_{\text {fibril }}$ of $12.6 \mathrm{GPa}$.

The relationship between the modulus of the composites and the volume fraction of the filler is approximately linear (Fig. 13, $\mathrm{R}^{2}=0.9$ ), in agreement

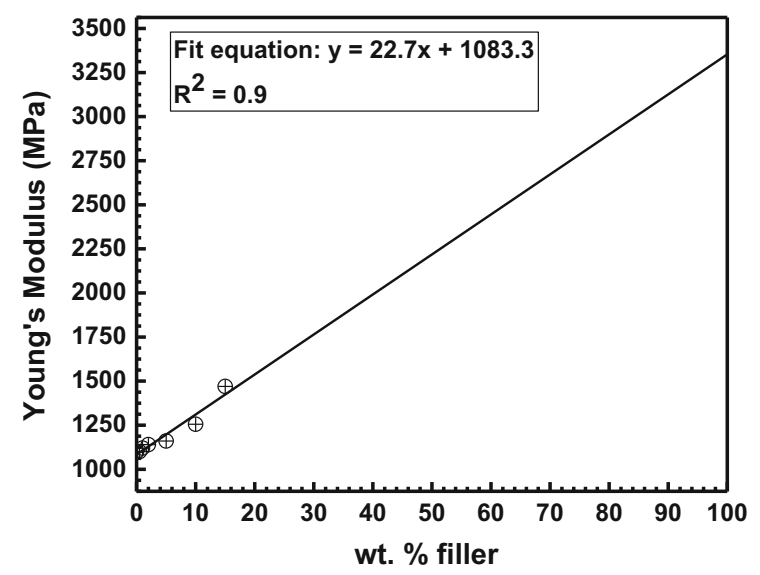

Fig. 13 Experimental Young's modulus data obtained from MFC-TA-C ${ }_{18}$ reinforced composites (dots). Solid line is a linear fit to these data, from which the fibril modulus $\mathrm{E}_{\text {fibril }}$ is estimated to be $12.6 \mathrm{GPa}$ 
with the rule of mixtures. A better dispersion of the cellulosic filler should be achieved before it becomes possible to obtain enhanced tensile and impact properties in the MFC reinforced composites, and reinforcement above a relatively low volume fraction.

\section{Conclusions}

In this study, a hydrophobic form of MFC was obtained by reacting an undried MFC slurry with tannic acid and octadecylamine. Sheets made from the product (MFC-TA- $\mathrm{C}_{18}$ ) were shown to have a low free surface energy, which favours the dispersion of the MFC in apolar polymers. Composites of MFC-TA- $\mathrm{C}_{18}$ in PPPE had higher modulus than the pure PPPE polymer when the loading of the MFC-TA- $\mathrm{C}_{18}$ was greater than $2 \mathrm{wt} \%$. Despite the effective dispersion of a large portion of the MFC-TA- $\mathrm{C}_{18}$ filler, the presence of some remaining aggregates impaired the mechanical performance of the composites, especially their impact properties, which were compromised at reinforcement loadings greater than $1 \mathrm{wt} \%$.

Filler dispersion in the composites plays a central role in the effectiveness of the reinforcement. In this study, spectroscopic and confocal microscopy techniques were utilised to determine the level of dispersion in the produced specimens. EDX images of composites reinforced with MFC-TA- $\mathrm{C}_{18}$ and MAgPP-MFC revealed the presence of large (up to $100 \mu \mathrm{m})$ and small aggregates in the composites. Due to the lack of selectivity of the EDX technique towards cellulose, it was impossible to determine whether the cellulose signal in the background was due to the presence of cellulose or to other contaminants.

ToF-SIMS imaging and SCLSM techniques were also used to determine the filler dispersion and agglomerate size in the reinforced composites. ToFSIMS analysis produced a 2D map of the $5 \mathrm{wt} \%$ MFCTA- $\mathrm{C}_{18}$ reinforced composites. ToF-SIMS results indicated that, despite the presence of large aggregates, a significant proportion of the surface-treated cellulose fibrils were dispersed at a much smaller scale. SCLSM findings are in accordance with ToFSIMS: the modification of MFC with TA- $\mathrm{C}_{18}$ improved the filler dispersion within the apolar PPPE composite. This finding is supported by the polymer background intensity signal increase when TA- $\mathrm{C}_{18}$ surface treatment was used and a decrease in the observed aggregate area. Increasing the MFC-TA- $\mathrm{C}_{18}$ loading to $15 \mathrm{wt} \%$ in the composite does not result in increased aggregation. Therefore, the composite is not saturated below a MFC-TA- $\mathrm{C}_{18}$ loading of $15 \mathrm{wt} \%$. The filler dispersion data obtained from SCLSM cluster analysis correlates well with the stiffness increases registered for the composites and with the sharp decrease in the impact properties. The critical aggregate size calculated for the MFC-TA- $\mathrm{C}_{18}$ reinforced polyolefin system is about $37 \mathrm{~nm}$. Because most of the aggregates found in the composite were greater than this value, regardless of the filler loading, and because of the imperfect nature of the cellulose aggregates, the real potential of the surface treated MFC reinforcement was not fully achieved. Further work is necessary to achieve a better level of filler dispersion and fully exploit the MFC potential as polyolefin reinforcement.

Acknowledgments This work was funded by the Engineering and Physical Sciences Research Council (Grant No. EP/L015 102/1) the Industrial Doctorate Centre (IDC) in Composites Manufacture.

Open Access This article is distributed under the terms of the Creative Commons Attribution 4.0 International License (http:// creativecommons.org/licenses/by/4.0/), which permits unrestricted use, distribution, and reproduction in any medium, provided you give appropriate credit to the original author(s) and the source, provide a link to the Creative Commons license, and indicate if changes were made.

\section{References}

Bledzki AK, Reihmane S, Gassan J (1996) Properties and modification methods for vegetable fibers for natural fiber composites. J Appl Polym Sci 59:1329-1336

Bonini C, Heux L, Cavaillé JY et al (2002) Rodlike cellulose whiskers coated with surfactant: a small-angle neutron scattering characterization. Langmuir 18:3311-3314

Cox HL (1952) The elasticity and strength of paper and other fibrous materials. Br J Appl Phys 3:72-79

Duchemin BJC, Newman RH, Staiger MP (2009) Structureproperty relationship of all-cellulose composites. Compos Sci Technol 69:1225-1230

Ejima H, Richardson JJ, Liang K et al (2013) One-step assembly of coordination. Science 341:154-157

Fekete E, Molnár S, Kim G-M et al (1999) Aggregation, fracture initiation, and strength of $\mathrm{PP} / \mathrm{CaCO} 3$ composites. J Macromol Sci 38:885-899

Gauthier R, Joly C, Coupas AC et al (1998) Interfaces in polyolefin/cellulosic fiber composites: chemical coupling, 
morphology, correlation with adhesion and aging in moisture. Polym Compos 19:287-300

Gruber E, Granzow C (1996) Preparing cationic pulp by graft copolymerisation. 1. Synthesis and characterization. Papier 50:293

Guo C, Zhou L, Lv J (2013) Effects of expandable graphite and modified ammonium polyphosphate on the flame-retardant and mechanical properties of wood flour-polypropylene composites. Polym Polym Compos 21:449-456

Habibi Y, Lucia LA, Rojas OJ (2010) Cellulose nanocrystals: chemistry, self-assembly, and applications. Chem Rev 110:3479-3500

Hafrén J, Zou W, Córdova A (2006) Heterogeneous “organoclick" derivatization of polysaccharides. Macromol Rapid Commun 27:1362-1366

Henriksson M, Berglund LA (2007) Structure and properties of cellulose nanocomposite films containing melamine formaldehyde. J Appl Polym Sci 106:449-456

Herrick F, Casebier R, Hamilton J, Sandberg K (1983) Microfibrillated cellulose: morphology and accessibility. J Appl Polym Sci 37:797-813

Heux L, Chauve G, Bonini C (2000) Nonflocculating and chiralnematic self-ordering of cellulose microcrystals suspensions in nonpolar solvents. Langmuir 16:8210-8212

Hu Z, Berry M, Pelton R, Cranston E (2017) One-pot waterbased hydrophobic surface modification of cellulose nanocrystals using plant polyphenols. Sustain Chem Eng 5:5018-5026

Iwamoto S, Nakagaito ANNN, Yano H (2007) Nano-fibrillation of pulp fibers for the processing of transparent nanocomposites. Appl Phys A Mater Sci Process 89:461-466

Jager I, Fratzl P (2000) Mineralized collagen fibrils: a mechanical model with a staggered arrangement of mineral particles. Byophys J 79:1737-1746

Johns MA, Lewandowska A, Eichhorn SJ (2019) Rapid determination of the distribution of cellulose nanomaterial aggregates in composites enabled by multi-channel spectral confocal microscopy. Microsc Microanal 25:682-689

Kazayawoko M, Balatinecz JJ, Woodhams RT (1997) Diffuse reflectance Fourier transform infrared spectra of wood fibers treated with maleated polypropylenes. J Appl Polym Sci 66:1163-1173

Khalil HPSA, Davoudpour Y, Islam MN et al (2014) Production and modification of nanofibrillated cellulose using various mechanical processes: a review. Carbohydr Polym 99:649-665

Klemm D, Heublein B, Fink HP, Bohn A (2005) Cellulose: fascinating biopolymer and sustainable raw material. Angew Chem Int Ed 44:3358-3393

Klemm D, Schumann D, Kramer F et al (2006) Nanocelluloses as innovative polymers in research and application. Adv Polym Sci 205:49-96

Klemm D, Kramer F, Moritz S et al (2011) Nanocelluloses: a new family of nature-based materials. Angew Chem Int Ed 50:5438-5466

Lee H, Dellatore SM, Miller WM, Messersmith PBPB (2007) Mussel-inspired surface chemistry for multifunctional coatings. Science 318:426-431

Lee K-Y, Aitomäki Y, Berglund LA et al (2014) On the use of nanocellulose as reinforcement in polymer matrix composites. Compos Sci Technol J 105:15-27
Leitner J, Hinterstoisser B, Wastyn M et al (2007) Sugar beet cellulose nanofibril-reinforced composites. Cellulose $14: 419-425$

Ljungberg N, Cavaillí JY, Heux L (2006) Nanocomposites of isotactic polypropylene reinforced with rod-like cellulose whiskers. Polymer (Guildf) 47:6285-6292

Maldas D, Kokta BV (1994) Role of coupling agents on the performance of woodflour-filled polypropylene composite. Int J Polym Mater Polym Biomater 27(1-2):77-88

Malkapuram R, Kumar V, Singh Negi Y, Negi YS (2008) Recent development in natural fiber reinforced polypropylene composites. J Reinf Plast Compos 28:1169-1189

Matias MC, De La Orden MU, Gonzalez Sánchez C, Martinez Urreaga J (2000) Comparative spectroscopic study of the modification of cellulosic materials with different coupling agents. J Appl Polym Sci 75:256-266

Maya JJ, Rajesh A (2008) Recent developments in chemical modification and characterization of natural fiber-reinforced composites. Polym Polym Compos 16:187-207

Maya JJ, Sabu T (2008) Biofibres and biocomposites. Carbohydr Polym 71:343-364

Miao C, Hamad WY (2013) Cellulose reinforced polymer composites and nanocomposites: a critical review. Cellulose 20:2221-2262

Missoum K, Belgacem MN, Bras J (2013) Nanofibrillated cellulose surface modification: a review. Materials (Basel) 6:1745-1766

Peijs T, Garkhail S, Heijenrath R et al (1998) Thermoplastic composites based on flax fibres and polypropylene: influence of fibre length and fibre volume fraction on mechanical properties. Macromol Symp 127:193-203

Pöllänen M, Suvanto M, Pakkanen TT (2013) Cellulose reinforced high density polyethylene composites-morphology, mechanical and thermal expansion properties. Compos Sci Technol 76:21-28

Qiu W, Endo T, Hirotsu T (2006) Interfacial interaction, morphology, and tensile properties of a composite of highly crystalline cellulose and maleated polypropylene. J Appl Polym Sci 102:3830-3841

Sclavons M, Laurent M, Devaux J, Carlier V (2005) Maleic anhydride-grafted polypropylene: FTIR study of a model polymer grafted by ene-reaction. Polymer 46:8062-8067

Sehaqui H, Zhou Q, Ikkala O, Berglund LA (2011) Strong and tough cellulose nanopaper with high specific surface area and porosity. Biomacromolecules 12:3638-3644

Sileika TS, Barrett DG, Zhang R et al (2013) Colorless multifunctional coatings inspired by polyphenols found in tea, chocolate, and wine. Angew Chem Int Ed 52:10766-10770

Siro I, Plackett D, Siró I, Plackett D (2010) Microfibrillated cellulose and new nanocomposite materials: a review. Cellulose 17:459-494

Spence KL, Venditti RA, Rojas OJ et al (2011) A comparative study of energy consumption and physical properties of microfibrillated cellulose produced by different processing methods. Cellulose 18:1097-1111

Spoljaric S, Genovese A, Shanks RA (2009) Polypropylenemicrocrystalline cellulose composites with enhanced compatibility and properties. Compos Part A Appl Sci Manuf 40:791-799 
Takase S, Shiraishi N (1989) Studies on composites from wood and polypropylenes. II. J Appl Polym Sci 37:645-659

Wambua P, Ivens J, Verpoest I (2003) Natural fibres: can they replace glass in fibre reinforced plastics? Compos Sci Technol 63:1259-1264

Woodhams RT, Thomas G, Rodgers DK (1984) Wood fibers as reinforcing fillers for polyolefins. Polym Eng Sci 24:1166-1171

Zimmermann T, Pöhler E, Geiger T (2004) Cellulose fibrils for polymer reinforcement. Adv Eng Mater 6:754-761
Zimmermann T, Pöhler E, Schwaller P (2005) Mechanical and morphological properties of cellulose fibril reinforced nanocomposites. Adv Eng Mater 7:1156-1161

Publisher's Note Springer Nature remains neutral with regard to jurisdictional claims in published maps and institutional affiliations. 